\title{
CONTROL METABÓLICO Y PROLACTINA EN LA ENFERMEDAD MENTAL GRAVE. INTERVENCIONES ENFERMERAS
}

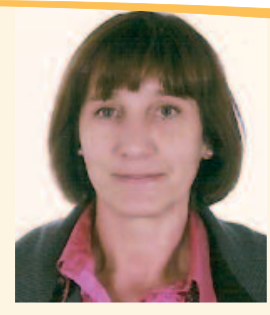

\section{M. ${ }^{a}$ LOURDES SÁNCHEZ PACHO}

Hospital Provincial de Ávila (SACYL).

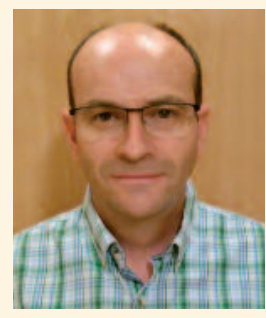

\section{JUSTO GONZÁLEZ PÉREZ}

Hospital Provincial de Ávila (SACYL).

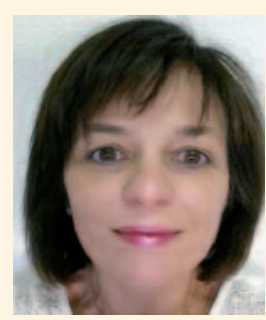

\section{M. ${ }^{a}$ DEL CARMEN MARTÍNEZ GUTIÉRREZ}

Centro de Salud Ávila Norte (SACYL).

\section{INTRODUCCIÓN}

Existe un amplio consenso sobre que los pacientes con enfermedad mental grave (EMG) tienen mayores tasas de morbimortalidad y más riesgo de muerte prematura en relación con enfermedades médicas —en especial, trastornos cardiovasculares-que la población general ${ }^{1}$. El síndrome metabólico (SM) se está convirtiendo en unos de los principales problemas de salud pública del siglo XXI, ya que se ha demostrado que aumenta en tres veces la prevalencia de la enfermedad cardiovascular que en el resto de la población ${ }^{2}$. No se conocen con claridad los mecanismos por los cuales algunos antipsicóticos (APS) pueden producir SM, puesto que no todos bloquean los mismos receptores ni tampoco en igual medida. La hiperprolactinemia (HPRL) es un efecto adverso frecuente provocado por los APS.
A pesar de su gravedad, con mucha frecuencia, estos efectos adversos son infradiagnosticados $y$, por consiguiente, infratratados, porque no se buscan de forma sistemática y no se objetivan externamente y el paciente siente vergüenza de referir algunos de ellos de manera espontánea ${ }^{3}$. El aumento de los niveles séricos de prolactina (PRL) puede provocar sufrimiento al paciente (disfunción sexual), ser estigmatizante (ginecomastia en varones), poner en riesgo la salud global y afectar a la funcionalidad y satisfacción con el tratamiento (repercutiendo en la adherencia) ${ }^{4}$. Resulta muy conveniente, tras los últimos trabajos publicados, conocer y evaIuar adecuadamente el alcance real de las consecuencias de la HPRL yatrogénica crónica por APS en el paciente con EMG, así como su posible abordaje clínico y terapéutico ${ }^{5}$. No se deben minimizar

Correspondencia: M. ${ }^{a}$ Lourdes Sánchez Pacho

Correo electrónico: lourdesspacho@enfermeriaavila.com 
los efectos sobre la sexualidad que producen algunos APS. No olvidemos que la sexualidad es un aspecto del ser humano, psicótico o no, que nos enriquece y nos hace más humanos ${ }^{6}$.

En este contexto, se plantea dentro de los equipos de salud mental (ESM) del Hospital Provincial de Ávila, perteneciente al Complejo Hospitalario de SACYL, como objetivo general, la necesidad de promover el cuidado de la salud física como parte integral del tratamiento de personas con EMG. Y como objetivos específicos: crear un sistema apropiado de monitorización de la salud física en estos pacientes; poner en marcha un protocolo de control y prevención del SM; monitorizar los niveles de PRL; mejorar la adherencia terapéutica; y aumentar la sensibilidad en estos aspectos entre los profesionales de la salud mental y atención primaria, así como entre pacientes y familiares.

\section{MATERIAL Y MÉTODO}

Se desarrolló un plan de trabajo con el objetivo de valorar la necesidad de implantar un programa para mejorar la salud física de los pacientes con EMG. Se realizaron varias reuniones de equipo para fijar los objetivos, los criterios de inclusión y el desarrollo del programa que respondiera a las necesidades de los pacientes y a los recursos del dispositivo asistencial.
Se realizaron búsquedas bibliográficas con el propósito de identificar intervenciones para mejorar la salud en estos pacientes. Se recopiló y seleccionó documentación, que fue la base para la elaboración de un programa de control de la salud física.

Se fijaron los criterios de inclusión en el programa: a) Pacientes con EMG que inician tratamiento con APS en seguimiento por los ESM. b) Pacientes en tratamiento con APS en seguimiento por los ESM con, al menos, uno de los siguientes factores de riesgo: hipertensión arterial; SM; HPRL; hipotiroidismo subclínico; aumento del índice de masa corporal superior a los límites de referencia; aumento del perímetro abdominal por encima de los valores de referencia.

Se elaboraron registros específicos informatizados, integrados en la historia clínica electrónica (JIMENA), que permitieron el acceso rápido a los datos recogidos y la monitorización continuada de los parámetros de salud física a lo largo del tiempo. Se realizaron controles de la salud física de los pacientes que cumplían los criterios de inclusión en el programa a través de la monitorización de los parámetros de control establecidos, tanto al inicio del tratamiento con APS, como, a los 3, 6, 12 y 24 meses. Se realizan valoraciones sobre antecedentes médicos y psiquiátricos personales y familiares; estado nutricional y nivel de actividad física; factores de riesgo de salud física: hábitos tóxicos, conductas sexuales de riesgo, enfermedades de transmisión sexual; revisión del tratamiento; control de síntomas o alteraciones conductuales; grado de adherencia terapéutica; efectos adversos de la medicación; SM, incluyendo aumento de peso, dislipidemia y diabetes; efectos extrapiramidales hormonales: tirotropina y PRL basal; efectos cardiovasculares; experiencias subjetivas que produzcan incomodidad en el paciente; y preocupaciones expresadas por el paciente o la familia.

\section{RESULTADOS}

Se consiguió el objetivo principal -ya que se puso en marcha el programa de salud física y, por ahora, lleva dos años de andadura-, así como los objetivos específicos, que sería extenso explicar en este formato, por lo que nos centraremos en el de «Monitorización de los niveles séricos de

\section{Se hace necesario el control y cuidado de la salud física como parte integral del tratamiento de personas con EMG.}


PRL», del cual surge un estudio observacional retrospectivo entre 2015 y 2017, incluyendo población rural y urbana, con individuos de ambos sexos, a partir de 18 años. De entre los 27044 pacientes que acuden a consultas externas de salud mental, se seleccionó a los que tenían un diagnóstico de EMG. Se incluye en el estudio a 202 pacientes, de los cuales, 122 son hombres $(H)$-el $60 \%$ y 80 son mujeres $(M)$-el $40 \%$ Estaban siendo tratados con un APS 103 y con más de un APS 99.

De los 103 tratados con monoterapia ( $57 \mathrm{H}$ y $46 \mathrm{M}$ ), presentaron HPRL 16 de ellos ( $8 \mathrm{H}$ y $8 \mathrm{M}$ ), que representan el $15,5 \%$ de la muestra, destacando que en ningún caso la HPRL era grave, pero sí moderada en un 56,25\% de ellos, correspondiéndose con $4 \mathrm{H}$

\section{Enfermería debe considerar el problema de la falta de adherencia como una prioridad y su evaluación ha de convertirse en una rutina.}

y $5 \mathrm{M}$, y leve en un $43,75 \%$ de los casos (4 H y $3 \mathrm{M}$ ) (fig. 1).

De los 99 tratados con politerapia (65 H y $34 \mathrm{M}$ ), presentaron HPRL 38 personas ( $15 \mathrm{H}$ y $23 \mathrm{M}$ ), un $38,8 \%$ de la muestra. Con politerapia, sí hubo casos de HPRL grave: un $15,7 \%$ de los pacientes, significándose que son $1 \mathrm{H} \mathrm{y}$ $5 \mathrm{M}$. Presentaron HPRL moderada un $50 \%$ de estos pacientes, en este caso, $6 \mathrm{H}$ y $13 \mathrm{M}$. Respecto a la HPRL leve, se detectó en un $34,3 \%$ de los casos, $8 \mathrm{H}$ y 5 $M$ (fig. 2).

Resumiendo los resultados, obtenemos que un $26,7 \%$ de los pacientes presentaron HPRL, un total de 54 pacientes. De ellos, el $70 \%$ con politerapia (38) y el $30 \%$ con monoterapia (16). En cuanto al sexo, el $43 \%$ eran hombres (23) y el $57 \%$ eran mujeres (31).

\section{CONCLUSIONES}

Se hace necesario el control y cuidado de la salud física como parte integral del tratamiento de personas con EMG. Resulta de gran utilidad contar con registros específicos informatizados. Los efectos metabólicos asociados al uso de APS son reversibles. En los

Figura 1. Niveles de gravedad de la hiperprolactinemia con monoterapia.

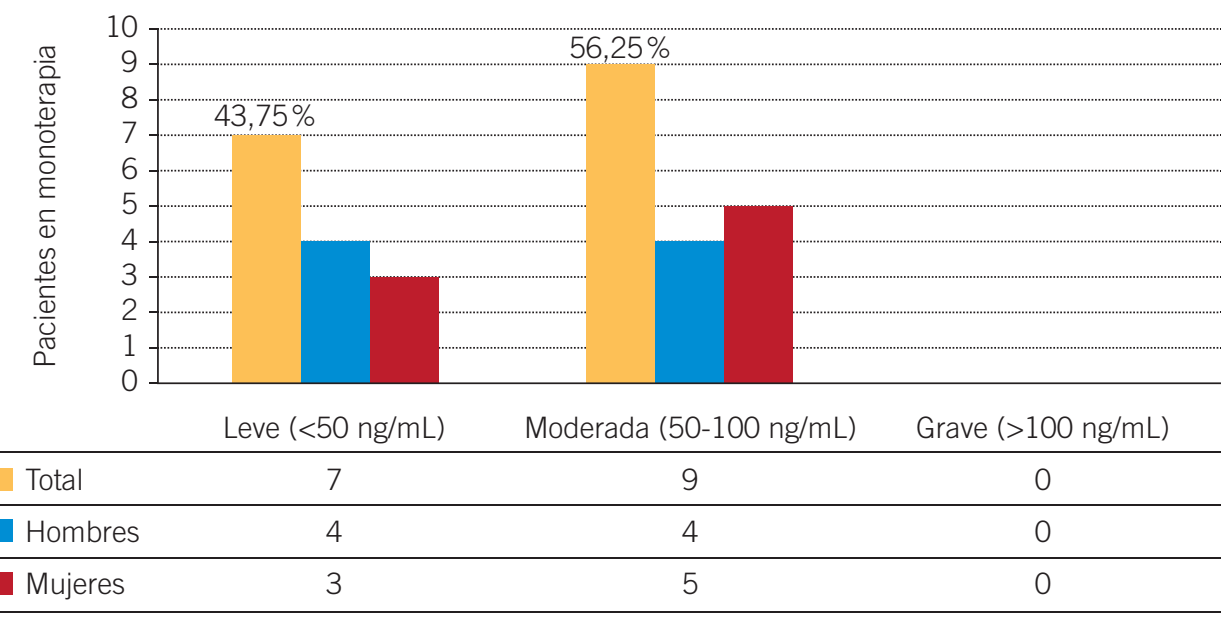


Figura 2. Niveles de gravedad de la hiperprolactinemia con politerapia.

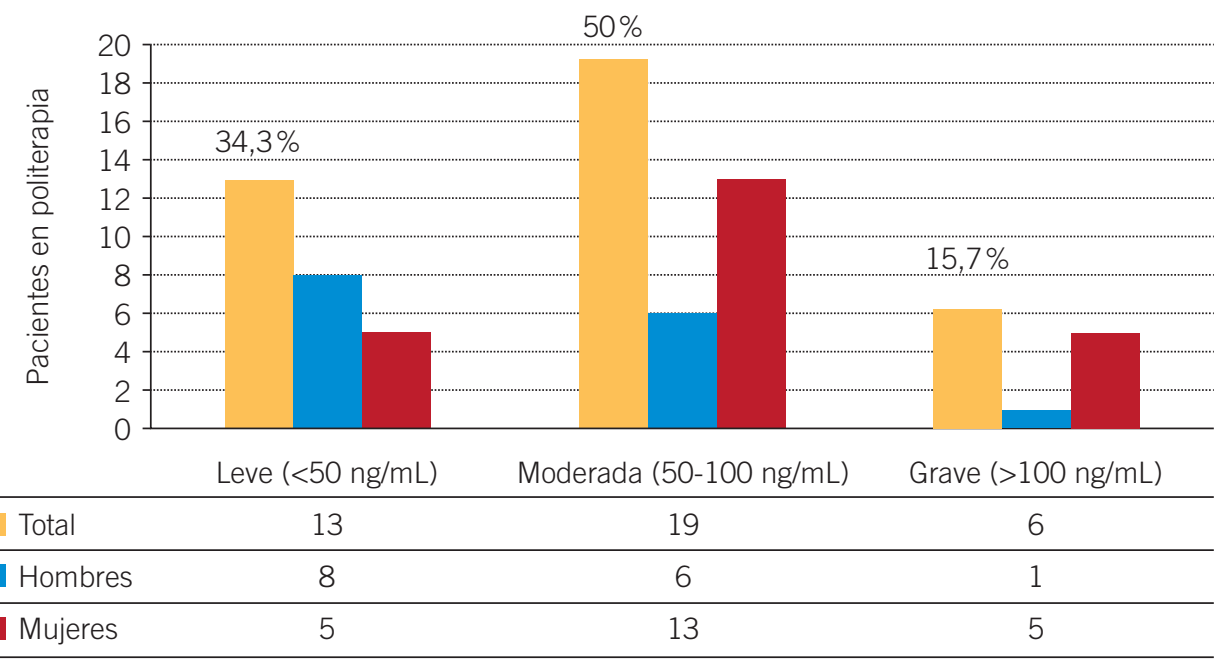

resultados obtenidos en el estudio que relaciona APS e HPRL, la administración de aripiprazol, en forma oral o inyectable, produce un significativo descenso de los niveles de PRL (fig. 3).
Previniendo la HPRL, se evitan los efectos que produce sobre la sexualidad y otras alteraciones

Figura 3. Tratamiento de la hiperprolactinemia (HPRL) durante el estudio.

\section{HPRL con monoterapia (16)}

Baja la PRL con aripiprazol (6)

Intolerancia al aripiprazol (1)

Baja la PRL (otras causas) (9)

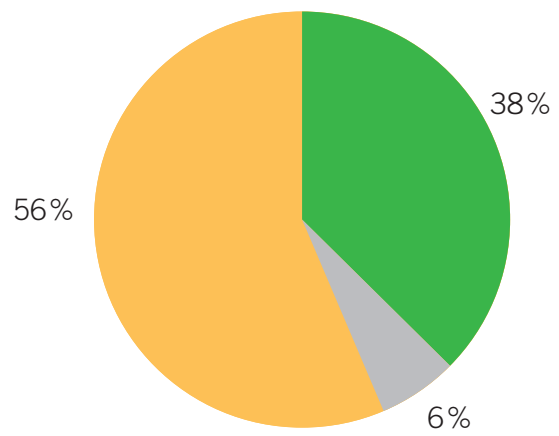

HPRL con politerapia (38)

Baja la PRL con aripiprazol (29)

Aumenta la PRL con aripiprazol (1)

No se añade aripiprazol (8)

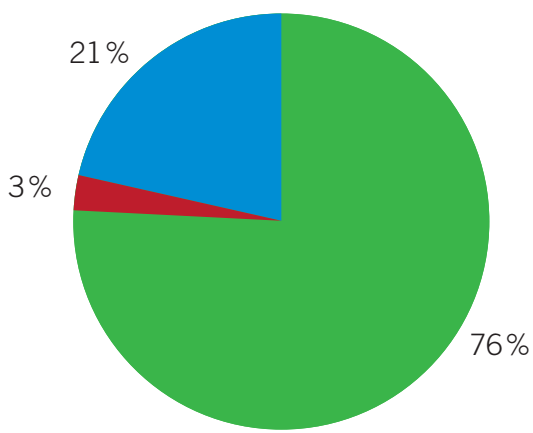

PRL: prolactina. 
metabólicas a corto, medio y largo plazo. Enfermería debe considerar el problema de la falta de adherencia como una prioridad y su evaluación ha de convertirse en una rutina. Los profesionales de enfermería pueden y deben integrar intervenciones para mejorar la salud física de estos pacientes.

\section{DISCUSIÓN}

Habría que realizar un estudio similar incluyendo el tipo de APS utilizado para poder valorar con más detalle la relación del fármaco empleado con la presentación de HPRL, si la politerapia incide en mayor medida en la aparición de la HPRL en mujeres y si la HPRL en mujeres se presenta con un nivel mayor de gravedad.

\section{BIBLIOGRAFÍA}

1. Gutiérrez-Rojas L, Azanza JR, Bernardo M, Rojo L, Mesa F, Martínez-Ortega JM. Prevalencia del síndrome metabólico en pacientes españoles con esquizofrenia y sobrepeso. El estudio CRESSOB. Actas Esp Psiquiatr. 2014;42(1):9-17.

2. Basterreche $N$, Zumárraga $M$, Arrue A, Olivas O, Dávila W. Aripiprazol como corrector de la hiperprolactinemia causada por paliperidona. Actas Esp Psiquiatr. 2012; 40(5):290-2.
3. Cortés Morales B. Síndrome metabólico y antipsicóticos de segunda generación. Rev Asoc Esp Neuropsiq. 2011;31(2):303-20.

4. Montejo AL, Arango C, Bernardo M, Carrasco JL, Crespo-Facorro B, Cruz JJ, et al. Consenso español sobre los riesgos y detección de la hiperprolactinemia iatrogénica por antipsicóticos. Rev Psiquiatr Salud Mental (Barc.). 2016;9(3): 158-73.

5. Montejo AL, Arango C, Bernardo M, Carrasco JL, Crespo-Facorro B, Cruz JJ, et al. Consenso multidisciplinar sobre las recomendaciones terapéuticas para la hiperprolactinemia iatrogénica secundaria a antipsicóticos. Front Neuroendocrinol. 2017;45:25-34.

6. Montejo AL. Esquizofrenia y sexualidad. Rev Enferm Salud Ment. 2017;6:3-4. 